\title{
Welcome to Instruments-A New Leading Journal for Advanced Research in Instrumentation
}

\author{
Antonio Ereditato \\ Albert Einstein Center for Fundamental Physics, Laboratory for High Energy Physics, University of Bern, \\ Sidlerstrasse, 5 CH-3012 Bern, Switzerland; antonio.ereditato@cern.ch; Tel.: +41-31-6318566 \\ Received: 24 August 2016; Accepted: 24 August 2016; Published: 27 August 2016
}

Instruments is a new MDPI peer-reviewed, open access journal focused on scientific instrumentation and on the related methods and theory. MDPI has just celebrated 20 years of publishing experience, with an impressive score of more than 160 journals, many of them have already been covered by the Science Citation Index (Web of Science) and Scopus. Instrumentation in scientific research must necessarily be one of the flagship themes of such a broad portfolio.

The main editorial objective for Instruments is to provide a forum for advanced research on instruments, experimental apparatus, techniques and data processing for fundamental science and its applications. Our ambition is to select high quality scientific papers presenting truly innovative results and major achievements in instrumentation for various research fields, such as physics, engineering, chemistry, material science, biology, medicine, etc. This broad spectrum of disciplines should naturally promote the submission of interdisciplinary and trans-disciplinary papers, as well.

Needless to say, instrumentation is the main tool of modern experimental science. Advances in instrumentation directly and immediately provide new research opportunities and open the way to real scientific innovation. As a physicist, I would like to mention that only thanks to the major breakthroughs in particle detectors, accelerator technologies, and space instrumentation in the last few decades, have we been able to make the recent outstanding discoveries in particle and astroparticle physics, astrophysics and cosmology. On the other hand, the use of the same basic techniques has opened unexpected applications for society; the medical application of physics is just one example.

Instrumentation has for centuries been a collateral aspect of experimental research. Scientists had to develop the tools themselves for their experiments as a required part of the research work. In the last few years, we have observed a change of paradigm. Large groups dedicate their efforts and resources solely to the development of new devices, sensors, equipment and large infrastructures for advanced research. This is backed by dedicated theoretical and numerical studies, as well as the development of new experimental methodologies. We specifically wish to address these emerging communities in addition to the "usual" customers of present instrumentation journals.

This leads to a natural question: why another journal on scientific instrumentation? One could certainly answer that Instruments is "new" for the broad spectrum of disciplines it covers, including several different fields; it publishes regular research papers, communications, letters, short notes and reviews, experimental, theoretical and computational results in as much detail as required by the authors. There is no restriction on paper length or number of figures and tables. This is true, but in my opinion it is not the first ambition of the publisher and the main novelty of Instruments. The goal that the Editorial Board and I have set is to publish only high quality papers, which present undisputable elements of innovation and not merely incremental or very sectorial results.

Usually instrumentation journals publish highly specialized work, surely of good scientific merit, but sometimes of limited interest to a broad community, hence leading to a minor editorial impact. Instead, we will make sure that all aspects of really innovative research in instrumentation are covered by this new open access journal, thereby aiming at a broad interdisciplinary public. We are convinced that new insights in instrumentation from material science could be easily applied to research in 
chemistry; novel electronic sensors will soon play a role in biology, and advanced photo-detectors for astronomy could help in promoting future engineering applications. We want to provide a forum for these multiple communities as a whole, favoring the establishment of new trans-disciplinary approaches. For this reason the standard must be set very high.

Last but not least, the free access nature of Instruments will be beneficial for researchers working outside of the academic communities and for a general public potentially interested in the many, and sometimes unpredictable, applications of instrumentation.

In conclusion, on behalf of the Editorial Board, let me warmly invite my scientist colleagues to choose our journal and submit their high quality, innovative, groundbreaking papers. They will be subject to a rigorous peer-review procedure and, if accepted, published online within a short time. We see Instruments as an exciting new journal and are fully committed to lead it to a position in which it will soon be recognized for the dissemination of cutting edge research.

Conflicts of Interest: The author declares no conflict of interest.

(C) 2016 by the author; licensee MDPI, Basel, Switzerland. This article is an open access article distributed under the terms and conditions of the Creative Commons Attribution (CC-BY) license (http://creativecommons.org/licenses/by/4.0/). 